\title{
Synthesizing scanning-mode acquisition in full-wave modelling of OCT
}

Callum M. Macdonald, Peter R. T. Munro

Callum M. Macdonald, Peter R. T. Munro, "Synthesizing scanning-mode acquisition in full-wave modelling of OCT," Proc. SPIE 11078, Optical Coherence Imaging Techniques and Imaging in Scattering Media III, 1107806 (19 July 2019); doi: 10.1117/12.2526812 


\title{
Synthesizing scanning-mode acquisition in full-wave modelling of OCT
}

\author{
Callum M. Macdonald and Peter R. T. Munro \\ Department of Medical Physics and Biomedical Engineering, University College London, UK
}

\begin{abstract}
"Full-wave" models of OCT imaging systems based on Maxwell's Equations are beginning to emerge as feasible alternatives to well established approximate models. These models are however computationally taxing when dealing with large samples. Here, in an effort to reduce the computational burden, we present a means of synthesizing full-wave scanning-mode OCT image formation using only a single simulation of a plane wave incident on a given sample medium. The method involves an angular spectrum approach which synthesizes the signal for arbitrary scan positions, and arbitrary illumination conditions. This is demonstrated to reduce the required simulation time by an order of magnitude when synthesizing OCT B-scans, as compared to previous methods which require a separate simulation for each scan position.
\end{abstract}

Keywords: Optical Coherence Tomography, Microscopy, Electromagnetic modelling.

\section{INTRODUCTION}

When modelling spectral-domain OCT image formation, we require a means of computing the interaction of a broadband source-field with an arbitrary sample, and specifically, the resulting scattered field. Given the complexity of this problem, approximate methods involving either first-order Born approximations, or stochastic Monte Carlo approaches have seen wide-spread employment. ${ }^{1,2}$ Alternatively, it is possible to acheive a full Maxwell's Equations based solution for the scattered field using Finite Difference Time Domain (FDTD), or Psuedo-Spectral Time Domain (PSTD) methods, but only recently has this become feasible for large samples (of meaningful size in a biomedical context). In this work we utilize a previously documented PSTD $\operatorname{code}^{3}$ for computing the scattered field responsible for forming our signal.

When modelling image formation for an OCT system, a typical operation of this PSTD code for a large sample would involve multiple simulations, one for each scan-position of the illuminating beam. In this work, we simplify the image formation model by performing only one PSTD simulation of the entire medium, instead of one simulation for each scan position. To do this we use the PSTD simulation to calculate the scattered field in the back-focal plane of an objective which results from plane-wave illumination of the entire sample. Then, as a post-process we use this data to synthesize the field that would have been observed from focused illumination at each scan position. These reconstructed fields are then used to compute the signal coupled into the sample arm of an OCT interferometer for each "scan position". This method reduces the required computation time of the PSTD code by an order of magnitude when synthesizing OCT B-scans, and has the advantage of being able to recompute the OCT signal for arbitrary source/detector functions without needing to re-run the PSTD code.

\section{SCANNING-MODE SIGNAL SYNTHESIS}

In Figure 1 we display the geometry of the problem. In Fig. 1(a), a plane wave travelling in the $+z$ direction $(\exp (i k z))$ illuminates a sample, and a fixed objective lens of focal length $f_{2}$ projects the scattered field onto its back-focal plane, resulting in a field on the dashed $x-y$ plane which we will call $g_{1}(x, y)$, where $x, y$ are fixed co-ordinates of the back-focal plane. Now considering a case where a plane wave illuminates the sample from a different angle (Fig. 1b), with directional components $k_{x}, k_{y}$, and where a displaced lens then projects the

Send correspondence to P. Munro: p.munro@ucl.ac.uk 
(a)

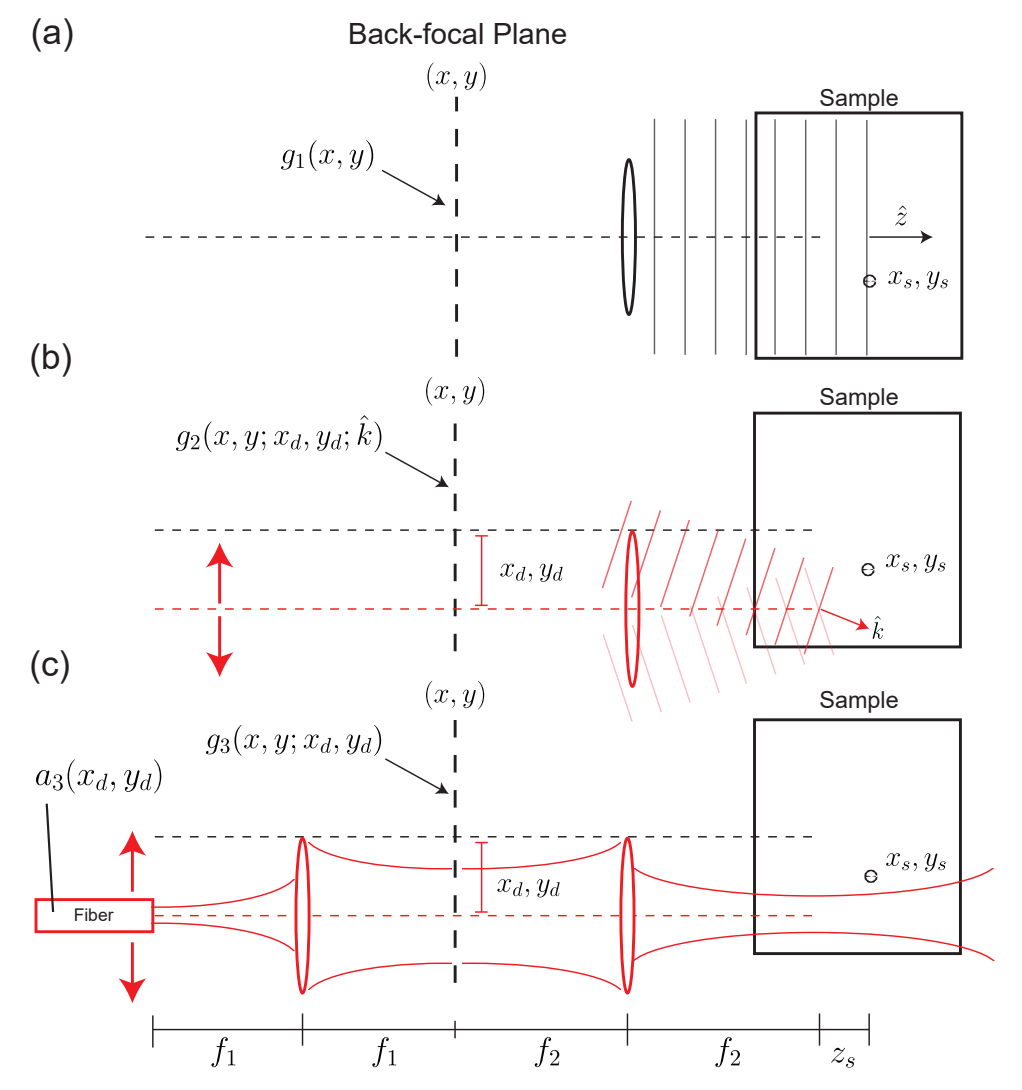

(b)

Figure 1. Details of three configurations under consideration. Red color highlights aspects of the system that undergo translation during the scanning process which we are attempting to replicate.

scattered field onto the back-focal plane, then for a point-like scatterer positioned at $\left(x_{s}, y_{s}, z_{s}\right)$, the fields in cases (a) and (b) can be shown to be related by:

$$
\begin{aligned}
g_{2}\left(x, y ; x_{d}, y_{d} ; k_{x}, k_{y}\right) & =g_{1}\left(\bar{x}+\frac{k_{x} f_{2}}{k}, \bar{y}+\frac{k_{y} f_{2}}{k}\right) \\
& \times \exp \left[\frac{i z_{s}}{f_{2}}\left(k_{x} \bar{x}+k_{y} \bar{y}\right)\right] \\
& \times \exp \left[-\frac{i k}{f_{2}}\left(x_{d} \bar{x}+y_{d} \bar{y}\right)\right] \\
& \times \exp \left[-i k_{x} x_{d}-i k_{y} y_{d}\right] .
\end{aligned}
$$

where $x_{d}, y_{d}$ is the displacement/scan position of the objective lens, and we have used the substitutions $\bar{x}=x-x_{d}$, and $\bar{y}=y-y_{d}$. Note that while in practice, scanning is usually achieved with the use of galvo-mirrors, we assume here that this is equivalent to physically translating the lens and source. It can be seen from the above expression that the field in case (b) is almost a simple translation of the field in the first case (a), but where the extra phase factors only disappear if the scatterer is in focus, and if the scan position is zero $\left(x_{d}=y_{d}=0\right)$. However, the extra factors have no dependence on the lateral position of the scatterer. Note that the depth of the scatterer $z_{s}$ does appear in the equation, however this term is slowly varying, which means that the synthesized signal will be accurate in the vicinity of the particular value of $z_{s}$ used, which may be changed to image different axial layers of the medium.

If we now consider the final case shown in Fig. 1(c), where a focused beam is illuminating the sample at a displaced scan position, we can use the result above, along with the angular spectrum of the source field to 
describe the resulting field projected onto the back-focal plane. For example, with a Gaussian illumination beam, we have:

$$
g_{3}\left(x, y ; x_{d}, y_{d}\right)=\int_{-\infty}^{\infty} g_{2}\left(x, y ; x_{d}, y_{d} ; k_{x}, k_{y}\right) \exp \left[-\frac{w_{0}^{2}}{4}\left(k_{x}^{2}+k_{y}^{2}\right)\right] d k_{x} d k_{y}
$$

Finally, the complex amplitude of the light coupled into the sample arm of the OCT system, $a_{3}$, can be found from the inner product of the collection mode, $\phi_{f}$, and the scattered field (taken in the back-focal plane). Remembering that the collection mode is also translating in tandem with the illumination mode (as these are usually from the same optical fiber), we can write:

$$
a_{3}\left(x_{d}, y_{d}\right)=\int_{-\infty}^{\infty} \phi_{f}\left(x-x_{d}, y-y_{d}\right) g_{3}\left(x, y ; x_{d}, y_{d}\right) d x d y
$$

Thus, if we first compute the back-focal plane field in the plane-wave illumination case from a single run of the PSTD code, $g_{1}(x, y)$, we can mimic the coupled amplitude of the focused scanning case for each scan position of interest, $a_{3}\left(x_{d}, y_{d}\right)$. This process is repeated for each of the wavenumbers included in the output of the PSTD code, taking $g_{1}(x, y ; k) \rightarrow a_{3}\left(x_{d}, y_{d} ; k\right)$, which can then be interfered with a reference field and Fourier transformed to produce a full 3D-OCT image of the sample.

\section{RESULTS AND CONCLUSIONS}

Figure 2(a) shows an assembly of scattering particles (in the shape of the letter "B') and in Figure 2(b) we show a simulated B-scan, computed from 120 separate PSTD simulations (one for each lateral scan position). In Figure 2(c) we show the synthesized OCT B-scan of the same medium, computed using only the data from a single PSTD simulation. It can be seen to closely resemble the reference B-scan. It is hoped that this order of magnitude reduction in computing requirements will aid in the development of these "full-wave" models of OCT image formation.
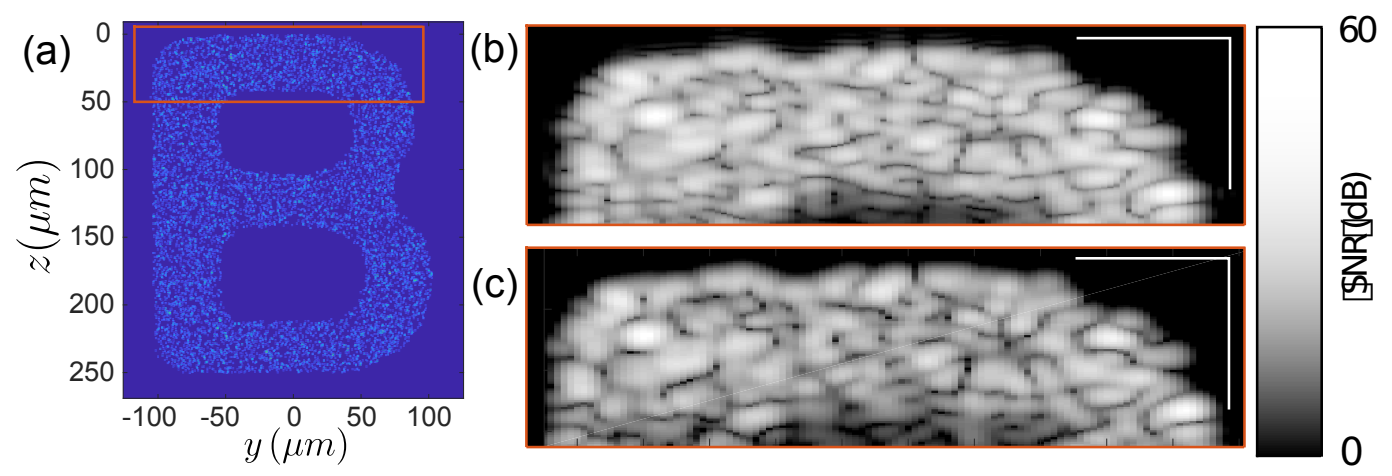

Figure 2. (a) Arrangement of scattering particles. (b) Reference OCT B-scan computed with 120 PSTD simulations (one for each scan position). (c) Synthesized OCT B-scan computed via the method described above (involving only a single PSTD simulation).

\section{REFERENCES}

[1] Ralston, T. S., Marks, D. L., Carney, P. S., and Boppart, S. A., "Inverse scattering for optical coherence tomography," JOSA A 23(5), 1027-1037 (2006).

[2] Yao, G. and Wang, L. V., "Monte carlo simulation of an optical coherence tomography signal in homogeneous turbid media," Physics in Medicine 63 Biology 44(9), 2307 (1999).

[3] Munro, P. R., "Three-dimensional full wave model of image formation in optical coherence tomography," Optics express 24(23), 27016-27031 (2016). 